Weinberg, E. D., SAz, A. K. \& Pilgren, E. Y. (1958). J. gen. Microbiol. 19, 419-433

\title{
Induction of Bacteriolysis by Cysteamine and Its Derivatives
}

\author{
By E. D. WEINBERG, A. K. SAZ AND ELISABETH Y. PILGREN \\ Department of Bacteriology, Indiana University, Bloomington, Indiana, and Section on \\ Medical and Physiological Bacteriology, National Institute of Allergy and Infectious \\ Diseases, National Institutes of Health, Bethesda, Maryland, U.S.A.
}

SUMMARY : Cysteamine and certain closely related derivatives induced lysis in a majority of 28 strains of Bacillus; cultures of all other genera tested were resistant. Forty-three additional sulphur and/or nitrogen containing compounds were unable to induce lysis. Optimum conditions for cysteamine activity included exposure of metabolizing post-log. phase organisms contained in shallow shaken layers of nutrient broth to the compound for 4-8 hr. Upon the completion of lysis, no formed cellular elements were visible by microscopy except flagella. The induction by cysteamine of a lysogenic bacteriophage or of an unusually large amount of an autolytic enzyme was not demonstrated.

Among the substances which provide some degree of protection to living cells subsequently exposed to ionizing radiation is a group of chemical compounds consisting of cysteamine ( $\beta$-mercaptoethylamine; MEA) and certain closely related derivatives. A preliminary report concerning the ability of the oxidized form of cysteamine (cystamine: 2,2-dithiobis-(ethylamine)) to induce lysis of organisms of various Bacillus spp. has been published (Weinberg, 1957). The present paper describes tests for induction of lysis with other members of the cysteamine group and with 43 related compounds. Various hypotheses concerning the mechanism of lytic induction are considered and the results of experiments designed to test some of these hypotheses are presented.

\section{METHODS}

Organisms. The test organisms (Table 1) were obtained from the collection of the Department of Bacteriology, Indiana University, except for the culture of Micrococcus lysodeikticus which was kindly supplied by Dr Arthur Schade, National Institutes of Health (Bethesda, Maryland, U.S.A.). Each strain was checked for purity by the usual cytological and cultural methods and was then maintained on nutrient agar (Difco) slopes. Subcultures on fresh nutrient agar slopes were made weekly.

Culture media. The majority of the experiments were performed with organisms grown in nutrient broth (Difco). In a few tests, specified in the section on results, a chemically defined medium consisting of the following ingredients, at the molarities $(\mathrm{M})$ indicated, dissolved in triple-distilled water was used: $1 \times 10^{-2}$, glucose; $1 \times 10^{-2}, \mathrm{NH}_{4} \mathrm{NO}_{3} ; 9 \times 10^{-3}, \mathrm{Na}_{2} \mathrm{HPO}_{4} ; 6 \times 10^{-3}$, $\mathrm{KH}_{2} \mathrm{PO}_{4} ; 3 \times 10^{-4}, \mathrm{MgSO}_{4} ; 2 \times 10^{-5}, \mathrm{FeSO}_{4} .7 \mathrm{H}_{2} \mathrm{O} ; \mathrm{pH}$ value, 7.0. Ten ml. 
quantities of the nutrient broth or defined liquid medium were added to $50 \mathrm{ml}$. Erlenmeyer flasks and sterilized by autoclaving.

Compounds tested for ability to induce lysis. The compounds tested for the ability to induce lysis or to affect lysis-inducing compounds are listed in Table 2. The compounds were dissolved in triple-distilled water and sterilized by autoclaving or, with the heat labile sustances, by filtration through sintered glass filters. Solutions of the compounds were added aseptically to flasks of inoculated culture medium at $(a)$ the beginning of the lag phase, or $(b)$ during the post-log. phase. When not in use, the solutions were stored at $4^{\circ}$; for some tests, however, fresh solutions of the cysteamine compounds were prepared daily.

Table 1. Susceptibility of test strains of Bacillus and other genera to induction of lysis by cysteamine

Cysteamine $\left(2 \times 10^{-3} \mathrm{M}\right)$ was added to post-log. phase cultures. Turbidity readings were made during the subsequent $24 \mathrm{hr}$. period of incubation. Of the sensitive strains, the majority of the $B$. subtilis group lysed within 4-8 hr.; the majority of the other groups required 8-24 hr. A dark pigment was produced from cysteamine by 1 sensitive and 1 resistant strain of $B$. megaterium and by 1 resistant strain of $B$. cereus.

\begin{tabular}{|c|c|c|c|c|}
\hline Genus & Species & $\begin{array}{l}\text { No. of } \\
\text { strains } \\
\text { tested }\end{array}$ & $\begin{array}{l}\text { No. of } \\
\text { resistant } \\
\text { strains }\end{array}$ & $\begin{array}{l}\text { No. of } \\
\text { sensitive } \\
\text { strains }\end{array}$ \\
\hline \multirow[t]{9}{*}{ Bacillus } & subtilis & 7 & $\mathbf{0}$ & 7 \\
\hline & megaterium & 6 & 1 & 5 \\
\hline & cereus & 4 & 1 & 3 \\
\hline & macerans & 2 & 1 & 1 \\
\hline & anthracis & 2 & 1 & 1 \\
\hline & sphaericus & 1 & $\mathbf{0}$ & 1 \\
\hline & brevis & $\mathbf{1}$ & $\mathbf{1}$ & $\mathbf{0}$ \\
\hline & circulans & 1 & $\mathbf{1}$ & $\mathbf{0}$ \\
\hline & unidentified & 4 & 3 & 1 \\
\hline Sarcina & lutea & 1 & 1 & $\mathbf{0}$ \\
\hline Staphylococcus & aureus & 1 & 1 & $\mathbf{0}$ \\
\hline Micrococcus & lysodeikticus & $\mathbf{1}$ & $\mathbf{1}$ & $\mathbf{0}$ \\
\hline Mycobacterium & avium & $\mathbf{1}$ & 1 & $\mathbf{0}$ \\
\hline Escherichia & coli & 1 & 1 & $\mathbf{0}$ \\
\hline Salmonella & typhimurium & 1 & 1 & $\mathbf{0}$ \\
\hline
\end{tabular}

Inocula and conditions of incubation. Flasks of nutrient broth or defined liquid medium were inoculated with organisms from the agar slopes and were shaken in a New Brunswick shaker (160 strokes/min.) at $37^{\circ}$. After $16 \mathrm{hr}$., the cultures were diluted in fresh nutrient broth or defined medium so that $1 \mathrm{ml}$. of the diluted culture contained approximately $2 \times 10^{6}$ viable organisms. Onetwentieth ml. portions of the diluted culture were added to the flasks to be used in the actual experiments. The experimental flasks were placed in the shaker and incubated at $37^{\circ}$ for periods from 1 to 6 days.

Determination of bacterial multiplication and lysis. The organisms of the inocula were observed to multiply and, in some cases, subsequently to lyse by turbidimetric measurements made with the Junior Coleman spectrophotometer model $6 \mathrm{~A}$ (at $660 \mathrm{~m} \mu$ ). To confirm multiplication and subsequent lysis 
Table 2. Compounds tested for ability to induce lysis of Bacillus subtilis strain Marburg

Each compound was added to $16 \mathrm{hr}$. nutrient broth cultures to obtain a final concentration of $1 \times 10^{-3} \mathrm{M}$. Turbidity readings were made at $16 \mathrm{hr}$. and at appropriate intervals up to $40 \mathrm{hr}$. of incubation.

I. Substituted ethanes

Ethyl amine

Ethylene diamine

Thio ethane

Cysteamine

Taurine

Ethanol

Ethylene chlorohydrin

Ethanol amine

$\beta$-Mercaptoethanol

Acetic acid

$$
\begin{aligned}
& \mathrm{CH}_{3} \cdot \mathrm{CH}_{2} \cdot \mathrm{NH}_{2} \\
& \mathrm{H}_{2} \mathrm{~N} \cdot \mathrm{CH}_{2} \cdot \mathrm{CH}_{2} \cdot \mathrm{NH}_{2} \\
& \mathrm{CH}_{3} \cdot \mathrm{CH}_{2} \cdot \mathrm{SH} \\
& \mathrm{H}_{2} \mathrm{~N} \cdot \mathrm{CH}_{2} \cdot \mathrm{CH}_{2} \cdot \mathrm{SH} \\
& \mathrm{H}_{2} \mathrm{~N} \cdot \mathrm{CH}_{2} \cdot \mathrm{CH}_{2} \cdot \mathrm{SO}_{3} \mathrm{H} \\
& \mathrm{CH}_{3} \cdot \mathrm{CH}_{2} \cdot \mathrm{OH} \\
& \mathrm{Cl} \cdot \mathrm{CH}_{2} \cdot \mathrm{CH}_{2} \cdot \mathrm{OH} \\
& \mathrm{H}_{2} \mathrm{~N} \cdot \mathrm{CH}_{2} \cdot \mathrm{CH}_{2} \cdot \mathrm{OH} \\
& \mathrm{HS}^{-} \mathrm{CH}_{2} \cdot \mathrm{CH}_{2} \cdot \mathrm{OH} \\
& \mathrm{CH}_{3} \cdot \mathrm{COOH}
\end{aligned}
$$

II. Substituted acetic acids

Thiolacetic acid Thioglycollic acid Sulphoacetic acid Glycine

$$
\begin{aligned}
& \mathrm{CH}_{3} \cdot \mathrm{COSH} \\
& \mathrm{HS} \cdot \mathrm{CH}_{2} \cdot \mathrm{COOH} \\
& \mathrm{HO} \cdot \mathrm{CH}_{2} \cdot \mathrm{CH}_{2} \cdot \mathrm{SO}_{3} \mathrm{H} \\
& \mathrm{H}_{2} \mathrm{~N} \cdot \mathrm{CH}_{2} \cdot \mathrm{COOH}
\end{aligned}
$$

III. Substituted propionic acids

L-Alanine
DL-Serine
$\beta$-Alanine
Mercaptopropionic acid
L-Cysteine
L-Cysteic acid
D-Penicillamine

$$
\begin{aligned}
& \mathrm{CH}_{3} \cdot \mathrm{CH}\left(\mathrm{NH}_{2}\right) \cdot \mathrm{COOH} \\
& \mathrm{HO} \cdot \mathrm{CH}_{2} \cdot \mathrm{CH}\left(\mathrm{NH}_{2}\right) \cdot \mathrm{COOH} \\
& \mathrm{H}_{2} \mathrm{~N} \cdot \mathrm{CH}_{2} \cdot \mathrm{CH}_{2} \cdot \mathrm{COOH} \\
& \mathrm{HS} \cdot \mathrm{CH}_{2} \cdot \mathrm{CH}_{2} \cdot \mathrm{COOH} \\
& \mathrm{HS} \cdot \mathrm{CH}_{2} \cdot \mathrm{CH}\left(\mathrm{NH}_{2}\right) \cdot \mathrm{COOH} \\
& \mathrm{HO}_{3} \mathrm{~S} \cdot \mathrm{CH}_{2} \cdot \mathrm{CH}\left(\mathrm{NH}_{2}\right) \cdot \mathrm{COOH} \\
& \mathrm{HS} \cdot \mathrm{C}\left(\mathrm{CH}_{3}\right)_{2} \cdot \mathrm{CH}\left(\mathrm{NH}_{2}\right) \cdot \mathrm{COOH}
\end{aligned}
$$

IV. Mono- and di- thio ethers

Thiodiethanol

Mercaptosuccinic acid

Aminoethyl sulphide

Cystamine

L-Cystine

L-Djenkolic acid

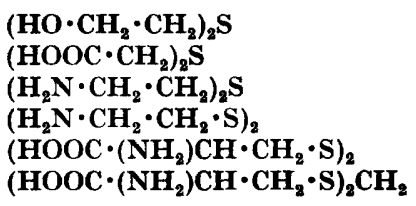

V. Substituted butyric acids

DL-Homocysteine DL-Methionine

$$
\begin{aligned}
& \mathrm{HS} \cdot \mathrm{CH}_{2} \cdot \mathrm{CH}_{2} \cdot \mathrm{CH}\left(\mathrm{NH}_{2}\right) \cdot \mathrm{COOH} \\
& \mathrm{H}_{3} \mathrm{C} \cdot \mathrm{S} \cdot \mathrm{CH}_{2} \cdot \mathrm{CH}_{2} \cdot \mathrm{CH}\left(\mathrm{NH}_{2}\right) \cdot \mathrm{COOH}
\end{aligned}
$$

VI. Isothiuronium compounds

Aminoethyl isothiuronium bromide (AET)

$\mathrm{S}, \gamma$-Aminopropyl isothiuronium bromide (APT) 
Table 2 (cont.).

VII. Pyrrolidine, thiazole, thiazoline, and thiazolidine compounds

L-Proline
2-Aminothiazole
Rhodanine
2-Methyl thiazoline
2-Mercapto thiazoline
Thiazolidine-4-carboxylic acid
2,4-Thiazolidine dione
2-Thiazolidine thione

L-Proline

2-Aminothiazole

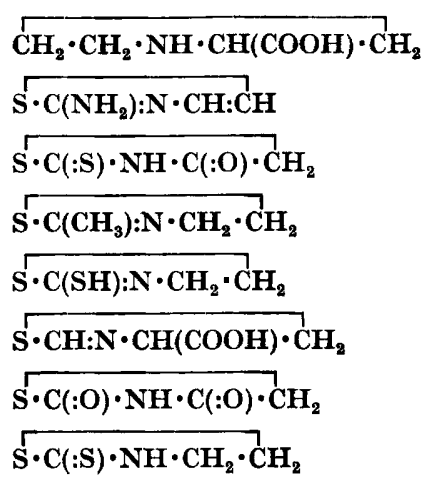

VIII. Miscellaneous compounds

Biotin

1,2-Diaminocyclohexane tetraacetic acid

Ethylene diamine tetraacetic acid

Glutathione

Nitrogen mustard

Pantetheine

Putrescine

Thiamine

Trypsin

as indicated by the increase and decrease in turbidity, plate counts of viable organisms and microscopic examinations were made of the cultures at appropriate intervals. The microscopic examinations included: Gram staining, cell-wall stain (Dyar, 1947), observation of wet mounts by phase microscopy, and photographs of organisms under electron microscopy.

Extraction of possible cysteamine-induced lytic principles. Organisms exposed to cysteamine added to the medium at the time of inoculation were ruptured by sonic oscillation or by ballotini in the Mickle tissue disintegrator at $6 \mathrm{hr}$., $10 \mathrm{hr}$., and $14 \mathrm{hr}$. after inoculation. Extracts of organisms not exposed to cysteamine were similarly prepared at the same times. The culture filtrates from 6,10 , and $14 \mathrm{hr}$. cysteamine-exposed and unexposed organisms were saved. Some cultures containing cysteamine were permitted to lyse and the lysates were saved for testing. In some cases, the culture filtrates and lysates were dialysed in a rocking dialyser against running tap water at $4^{\circ}$ for periods from $3 \mathrm{hr}$. to 4 days and the materials within the bag were saved for testing. The cell-free extracts, culture filtrates, lysates, and dialysis residue were stored at $-20^{\circ}$ when not in use.

Assay systems for detection of possible lytic principles. In general, organisms grown in nutrient broth for $16 \mathrm{hr}$. were used to detect lytic activity. The organisms were either kept in their culture medium or were removed by centrifugation and resuspended in the test solutions. In a few tests, 6 and $10 \mathrm{hr}$. crops of organisms were used. In experiments designed to study tem- 
perature effects, $16 \mathrm{hr}$. crops of organisms either retained in their culture medium or centrifuged and resuspended in $\mathrm{m} / \mathbf{3 0}$ phosphate buffer ( $\mathrm{pH} 8 \cdot 0$ ) were heated at various temperatures for $10 \mathrm{~min}$. immediately before being used in the assay system. On occasion, assay organisms were grown in the defined medium rather than in nutrient broth and were then either retained in their culture medium or were resuspended in staled nutrient broth. In addition to intact organisms, isolated cell walls were used as an assay tool. The method of cell-wall extraction and purification used was that of Salton \& Horne (1951).

\section{RESULTS}

Compounds which induced lysis of Bacillus subtilis strain Marburg Of the 48 compounds listed in Table 2 , only 5 were able to induce lysis under the conditions of the experiments (Table 1 ). The active substances included cysteamine and 4 closely related derivatives; the structural formulae of these compounds are given in Fig. 1. It should be noted that when aminoethyl isothiuronium bromide (AET) and S- $\gamma$-aminopropyl isothiuronium bromide

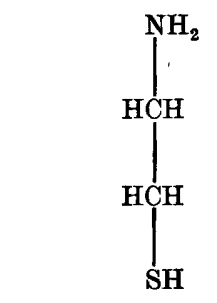
Cysteamine; $\beta$-mercaptoethylamine (MEA)

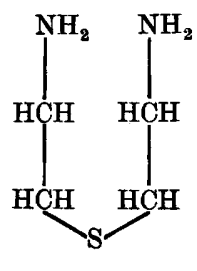

Aminoethyl sulphide

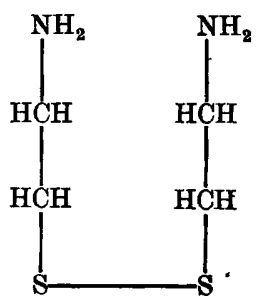

Cystamine;

2, 2'-dithiobis (ethylamine)<smiles>N=C([Nb])SC(CBr)C(N)Br</smiles>

Aminoethyl isothiuronium bromide (AET)<smiles>N=C([NH3+])SC(C(N)[NH3+])C(N)C(N)Br</smiles>

$\mathrm{S}, \gamma$-aminopropyl isothiuronium bromide (APT)

Fig. 1. Structural formulae of the compounds which induce lysis. 
(APT) are dissolved in neutral aqueous solutions, an intra-transguanylation occurs so that the active compounds are believed to be 2-mercaptoethylguanidine and 3-mercaptopropylguanidine, respectively (Shapira, Doherty \& Burnett, 1957).

The bacteriostatic concentration of each of the cysteamine compounds is indicated in Fig. 2 and the concentrations which induced lysis are presented in Fig. 3. It is apparent that sub-bacteriostatic concentrations were quite

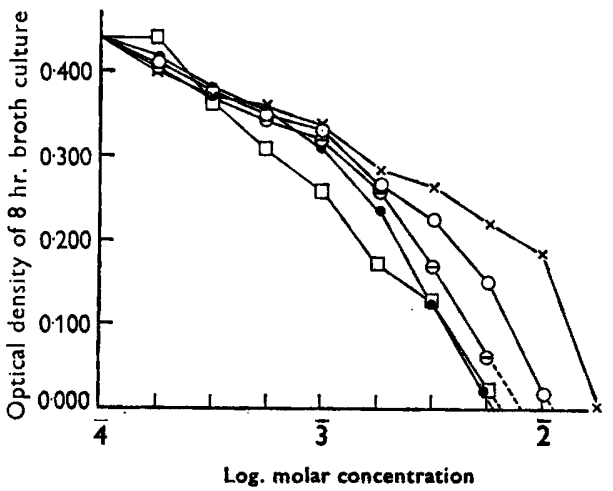

Fig. 2

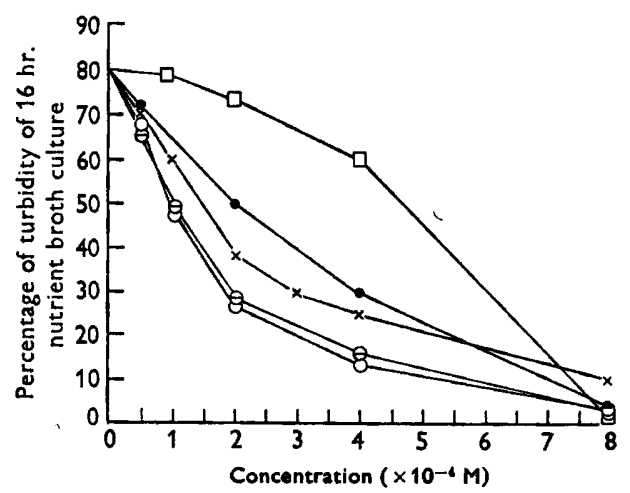

Fig 3

Fig. 2. Ability of various concentrations of the cysteamine compounds (added at the time of inoculation) to prevent growth of Bacillus subtilis strain Marburg in nutrient broth. Growth in the absence of the compounds was equivalent to that obtained in the presence of a concentration of $1 \times 10^{-4}$ M. $\square-\square$, APT; -0 , AET; $x-\times$, cysteamine; $\ominus-\ominus$, cystamine; $O-O$, amino ethyl sulphide.

Fig. 3. Ability of sub-bacteriostatic concentrations of the cysteamine compounds (added to $16 \mathrm{hr}$. nutrient broth cultures of Bacillus subtilis) to induce lysis. The readings were made at $24 \mathrm{hr} . \square-\square$, APT; - , AET; $x-x$, cysteamine; $\ominus-\Theta$, cystamine; $\bigcirc-O$, amino ethyl sulphide.

effective in induction of lysis. The time required for induction of lysis by various concentrations of cysteamine is given in Fig. 4. It may be noted that lysis occurred mainly between 4 and $8 \mathrm{hr}$. after the addition of the compound to post-log.-phase cultures. In the presence of suboptimal concentrations of cysteamine, a small amount of lysis occurred in the subsequent $16 \mathrm{hr}$. period; no additional cysteamine-induced lysis occurred thereafter.

\section{The spectrum of bacterial sensitivity to induction of lysis by cysteamine}

Of the test bacterial strains listed in Table 1, it may be observed that the majority of the strains of Bacillus especially within the species Bacillus subtilis, were susceptible to lytic induction. Attempts were made to induce lysis of strains of other genera by cysteamine by: (1) increasing the concentration of cysteamine to as much as $1 \times 10^{-2} \mathrm{M}$; (2) adjusting the $\mathrm{pH}$ value of post-log.-phase cultures to $8 \cdot 5$; (3) extending the time of contact between cysteamine and the strains to 6 days. These attempts to induce lysis were unsuccessful. 


\section{Physical and chemical environmental requirements for induction of lysis of Bacillus subtilis strain Marburg by cysteamine}

Despite the fact that sub-bacteriostatic concentrations of cysteamine induced lysis, such concentrations could be included in the culture medium at the time of inoculation without harm to the organisms of the inoculum. The organisms proceeded to complete the lag and logarithmic growth phases

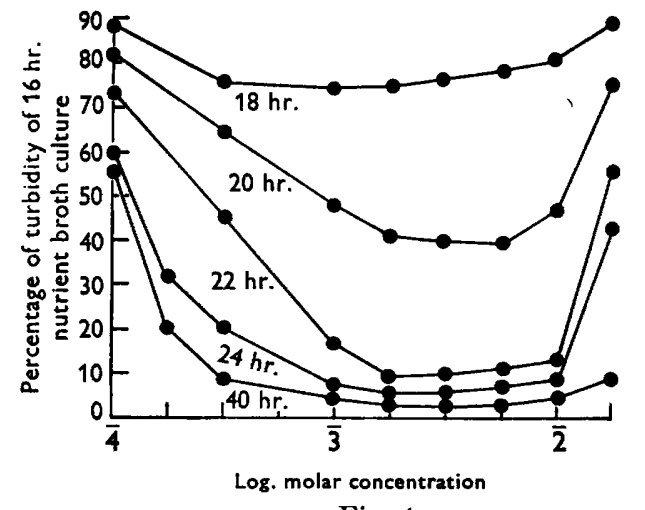

Fig. 4

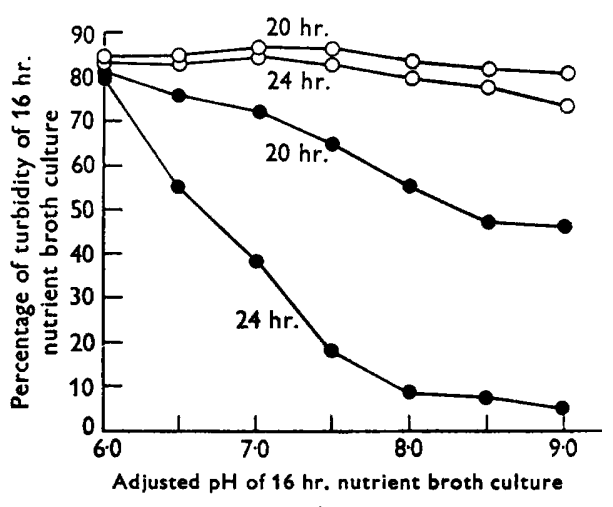

Fig. 5

Fig. 4. Ability of various concentrations of cysteamine (added to $16 \mathrm{hr}$. nutrient broth cultures) to induce lysis in Bacillus subtilis. In the absence of the compound, cultures decreased in turbidity to $70 \%$ of the $16 \mathrm{hr}$. turbidity at $40 \mathrm{hr}$.

Fig. 5. Effect of changing the $\mathrm{pH}$ value of $16 \mathrm{hr}$. nutrient broth cultures of Bacillus subtilis on the ability of cysteamine (added at the time of the $\mathrm{pH}$ adjustment) to induce lysis. The $\mathrm{pH}$ value of the $16 \mathrm{hr}$. cultures before adjustment was 8.7. $0-0$, no cysteamine; $1 \times 10^{-3} \mathrm{M}$ cysteamine.

of growth as rapidly as organisms in plain nutrient broth. The post-log. phase in each case was reached in approximately $10 \mathrm{hr}$.; in the cultures containing cysteamine, lysis occurred between 14 and $18 \mathrm{hr}$. Organisms in the control cultures retained their structural integrity for 6 days, at which time they were discarded. Cysteamine and aminoethyl sulphide could also be added either at the time of inoculation or during the post-log. period to obtain lysis, whereas the two isothiuronium compounds (AET, AEP) induced lysis only when they were added to post-log. phase cultures.

The influence of various physical and chemical environmental factors on induction of lysis by cysteamine is indicated in Table 3 and in Fig. 5. The most efficient conditions for such lysis included growth and maintenance of the cultures in nutrient broth in shaken shallow layers at $37^{\circ}$ without alteration of the normal post-log. $\mathrm{pH}$ value of 8.7 . Because of a deficiency of available manganese in nutrient broth (Weinberg, 1955) such cultures could not sporulate. Under conditions where sporulation occurs, as in nutrient agar, lysis induced by cysteamine required approximately 24-28 hr. rather than 4-8 hr. (Weinberg, 1957). It is probable that germination of the spores followed by 
logarithmic growth was required before the organisms became competent to lyse.

Attempts were made to suppress lysis in nutrient broth by increasing the molar strength of the environment with high concentrations of sucrose or glucose. As is indicated in Table 3, high concentrations of these carbohydrates caused both control and cysteamine-exposed organisms to lyse. Lower concentrations retarded the induction of lysis by cysteamine. The formation

Table 3. Effect of environmental conditions on ability of cysteamine to induce lysis of post-log. phase assay organisms of Bacillus subtilis strain Marburg

The organisms were grown in shaken shallow layers of nutrient broth (except in E) at $37^{\circ}$ for $16 \mathrm{hr}$. Cysteamine $\left(1 \times 10^{-3} \mathrm{M}\right)$ was added at $16 \mathrm{hr}$. and incubation was continued under the various test conditions for a subsequent period of $48 \mathrm{hr}$.

Condition during $48 \mathrm{hr}$. post-log. phase (A) Amount of
aeration

(B) Temperature

(C) $\mathrm{pH}$ value (see

Figure 5)

(D) Assay organisms grown in nutrient broth

(1) Shaken shallow layer

(2) Aspirated air

(3) Compressed air layer

(1) $80^{\circ}$

(2) $58^{\circ}$

(3) $37^{\circ}$

(4) $25^{\circ}$

(5) $2^{\circ}$

(1) 8-9

(2) $7-8$

(3) 6-7

(1) Resuspended in

(2) Resuspended in through deep layer through deep layer

(4) Stationary shallow Slow, partial nutrient broth filtrate defined medium filtrate (adjusted to pH 8.0)
(3) Resuspended in $\mathrm{M} / 30 \mathrm{PO}_{4}$ buffer (pH 8.0)

(E) Assay organisms (1) Resuspended in grown in defined medium nutrient broth filtrate

(2) Resuspended in defined medium filtrate (adjusted to pH 8.0)

(F) Increase in

(1) 1.4M-sucrose or glucose

(2) $0.7_{M}$-sucrose or glucose

(3) $0 \cdot 14 \mathrm{M}$-sucrose or glucose
Rapidity and extent of lysis

\begin{tabular}{|c|c|}
\hline $\begin{array}{l}\text { Control } \\
\text { organisms }\end{array}$ & $\begin{array}{c}\text { Cysteamine- } \\
\text { exposed organisms }\end{array}$ \\
\hline None & Rapid, complete \\
\hline None & Slow, partial \\
\hline None & Slow, partial \\
\hline Slow, partial & Slow, complete \\
\hline $\begin{array}{l}\text { None } \\
\text { Rapid, complete } \\
\text { None } \\
\text { None } \\
\text { None }\end{array}$ & $\begin{array}{l}\text { None } \\
\text { Rapid, complete } \\
\text { Rapid, complete } \\
\text { Slow, complete } \\
\text { None }\end{array}$ \\
\hline $\begin{array}{l}\text { None } \\
\text { None } \\
\text { None }\end{array}$ & $\begin{array}{l}\text { Rapid, complete } \\
\text { Slow, partial } \\
\text { Slow, slight }\end{array}$ \\
\hline None & Rapid, complete \\
\hline None & Slow, partial \\
\hline Slow, partial & Slow, complete \\
\hline None & Rapid, complete \\
\hline None & None \\
\hline Immediate, complete & Immediate, complete \\
\hline Rapid, partial & Rapid, complete \\
\hline None & Slow, complete \\
\hline
\end{tabular}


of protoplasts was not observed even when bovine serum albumin was added to the lysing preparations that were being examined by phase microscopy.

Of interest are the observations that a few of the $\mathbf{4 3}$ compounds which did not induce lysis partially suppressed the activity of cysteamine. The cysteamine-suppressing compounds included $\mathrm{D}$ - or L-cysteine, DL-cystine, Dpenicillamine, thiazolidine thione and thiazolidine-4-carboxylic acid. The last compound, an antimetabolite of proline, was the most efficient suppressor of cysteamine (Fig. 6); L-proline had no effect on the activity of thiazolidine-4carboxylic acid.

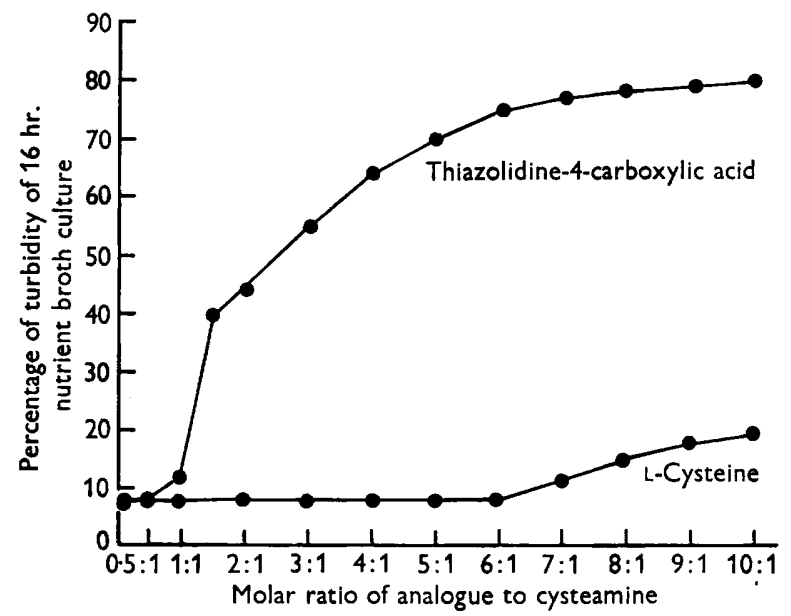

Fig. 6. Ability of thiazolidine-4-carboxylic acid and L-cysteine to suppress lysis-induction activity of cysteamine in Bacillus subtilis. The analogue and cysteamine were added at $16 \mathrm{hr}$. and the readings were made at $24 \mathrm{hr}$.

Various inorganic ions were tested for their effect on cysteamine-induced lysis. $\mathrm{Cu}^{++}$slightly accelerated lysis; $\mathrm{Mg}^{++}$and $\mathrm{MoO}_{4}^{-}$were inactive; $\mathrm{Fe}^{++}$, $\mathrm{Mn}^{++}$, and $\mathrm{Zn}^{++}$slightly delayed lysis; $\mathrm{Co}^{++}$and $\mathrm{Ni}^{++}$totally inhibited the cysteamine effect. In each case a concentration equimolar to cysteamine was used; since some of the metallic ions are bacteriostatic in these concentrations, both cysteamine and the ions were added to post-log. cultures rather than to fresh cultures.

Other requirements for cysteamine-induced lysis included: (1) metabolizing organisms rather than organisms inactivated by exposure to $80^{\circ}$ for $10 \mathrm{~min}$. or isolated cell walls; $(2)$ the continual presence of cysteamine (or a compound synthesized by cysteamine-exposed organisms) in the culture filtrate. When at any time during the period required for lysis to occur, the cysteamine-exposed organisms were removed from their culture medium and resuspended in normal culture filtrate of the same age, lysis was considerably slower and often incomplete.

\section{Presence of formed elements in the lysate}

As lysis proceeded to completion, the majority of organisms (as determined by successive stained preparations) first became Gram-negative and then 
disappeared. When organisms in lysing cultures were observed by phase microscopy, the organisms appeared to become less dense before they disappeared. No formed elements were detected in lysates by phase microscopy. Cell-wall stains of partially lysed cultures revealed circular 'ghosts' which were not evident in completely lysed preparations. Similarly, circular ghosts were seen in electron photomicrographs of partially-lysed cultures. Electron photomicrographs of normal cultures contained only intact organisms while those of completely lysed cultures contained no formed elements except flagella. Seemingly completely lysed cultures were, however, not sterile; plate counts to detect viable organisms indicated that a few hundred organisms $/ \mathrm{ml}$. lysate survived exposure to cysteamine. It is noteworthy that these few surviving organisms were not able to multiply in filtrates of cysteaminelysed cultures, whereas survivors of populations lysed by other means (such as high concentrations of sucrose) were usually able to undergo logarithmic growth in their homologous culture media when incubation was continued.

The ability of dialysed lysates, culture filtrates, and fresh nutrient broth to induce lysis (in the absence of cysteamine) of normal and heated assay organisms with and without added $\mathrm{Co}^{++}$

An extensive series of experiments was performed to determine whether cysteamine-exposed organisms produced a bacteriophage or an unusually large quantity of an autolytic enzyme. Tests for bacteriophage production consisted of: (a) adding successive crops of post-log. normal organisms to lysates, and $(b)$ the addition of lysates to normal organisms of various ages in nutrient broth and on nutrient agar. To detect the presence of autolytic enzymes, similar experiments were performed with soluble extracts of normal and cysteamine-exposed crops of organisms of various ages which had been ruptured by sonic oscillation and by Mickle disintegration. No evidence of lytic activity in any of these preparations was obtained except in those containing residual cysteamine. When cysteamine was present in the minimum concentration required for lysis of the first crop of organisms, subsequent crops were not lysed in the particular sample. When a twofold, threefold, fourfold, or fivefold concentration of cysteamine was contained in the original sample, two, three, four or five but not six crops of post-log. organisms, respectively, could be added successively to the sample and be completely lysed.

To remove residual cysteamine from cell-free extracts or from lysates, the samples were dialysed in a rocking dialyser against running tap water at $4^{\circ}$ for periods from $3 \mathrm{hr}$. to 4 days. Samples of culture filtrate and fresh nutrient broth were likewise dialysed for 2 days. Dialysed cell-free extracts possessed no lytic activity for normal post-log. assay organisms but lysates from cysteamine-exposed organisms did possess lytic activity when dialysed for at least 2 days. Normal culture filtrate and fresh nutrient broth which had been dialysed for 2 days similarly acquired lytic activity, although they were less efficient than dialysed lysates. The time required for lysis of $50 \%$ of crops of assay organisms added to the various samples is summarized in Table 4. It may 
be seen that as the residual cysteamine was removed from lysates by dialysis up to $24 \mathrm{hr}$. the lysates became somewhat less active but that activity was significantly greater in lysates dialysed for $48 \mathrm{hr}$. With some lysates, a period of dialysis of 3 days was required to obtain maximum activity; no additional activity of any sample was gained by a 4 th day of dialysis.

Table 4. Effect of dialysis of test samples and of heat and $\mathrm{Co}^{++}$activation on rapidity of lysis of post-log. phase assay organisms of Bacillus subtilis

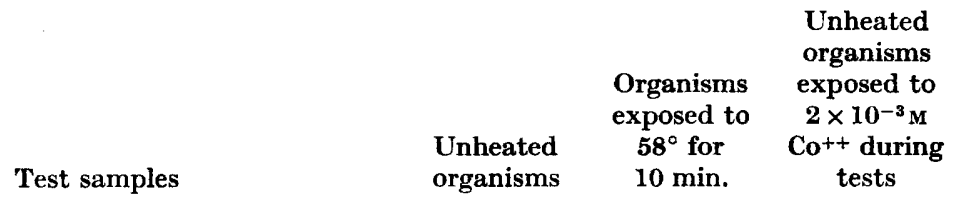

(A) Post-log. phase culture filtrates from which normal organisms were removed

(1) Non-dialysed

(2) Non-dialysed plus $8 \times 10^{-4} \mathrm{M}$-cysteamine

(3) Dialysed $48 \mathrm{hr}$.

\begin{tabular}{|c|c|c|}
\hline \multicolumn{3}{|c|}{ Time for $\mathbf{5 0} \%$ lysis (hr.) } \\
\hline No lysis & $1 \cdot 3$ & $2 \cdot 4$ \\
\hline $4 \cdot 0$ & 0.6 & 6.5 \\
\hline $12 \cdot 0$ & $1 \cdot 2$ & $\mathbf{3 \cdot 0}$ \\
\hline
\end{tabular}

(B) Post-log. phase lysate from cysteamine-exposed cultures

\begin{tabular}{|c|c|c|}
\hline $\begin{array}{l}\text { (1) Non-dialysed (contained slight amount } \\
\text { of residual cysteamine) }\end{array}$ & $13 \cdot 0$ & $1 \cdot 2$ \\
\hline (2) Dialysed $3 \mathrm{hr}$. & $15 \cdot 0$ & 1.0 \\
\hline (3) Dialysed $6 \mathrm{hr}$. & $16 \cdot 0$ & $\mathbf{1} \cdot \mathbf{1}$ \\
\hline (4) Dialysed $24 \mathrm{hr}$. & $16 \cdot 5$ & 1.0 \\
\hline (5) Dialysed $48 \mathrm{hr}$. & $8 \cdot 4$ & 0.5 \\
\hline $\begin{array}{l}\text { Fresh nutrient broth } \\
\text { (1) Non-dialysed } \\
\text { (2) Dialysed } 48 \mathrm{hr} \text {. }\end{array}$ & $\begin{array}{l}\text { No lysis } \\
\mathbf{2 4} \cdot \mathbf{0}\end{array}$ & $\begin{array}{l}\text { No lysis } \\
2 \cdot 1\end{array}$ \\
\hline
\end{tabular}

Since the autolytic enzyme of post-log.-phase organisms of Bacillus subtilis studied by Strange \& Dark (1957) was activated by exposure to $58^{\circ}$ and by the presence of high concentrations of $\mathrm{Co}^{++}$, it seemed of interest to include a study of the effect of these two conditions in lysis of $B$. subtilis by the test samples. Heat activation was accomplished by heating the assay organisms (in a small volume of $\mathrm{pH} 8.0$ phosphate buffer) for $10 \mathrm{~min}$. at $58^{\circ}$ before suspension of the organisms in the various test samples. The actual tests were then performed at $37^{\circ}$, although the results were similar when the tests were conducted at $58^{\circ}$. Activation by $\mathrm{Co}^{++}$was tested by adding to the test samples a solution of $\mathrm{CoSO}_{4} \cdot 7 \mathrm{H}_{2} \mathrm{O}$ to obtain a final concentration of $\mathrm{Co}^{++}$of $2 \times 10^{-3} \mathrm{M}$.

It can be observed in Table 4 that exposure of the normal post-log. phase assay organisms to $58^{\circ}$ markedly accelerated lysis in all samples. As was true of non-heated organisms, organisms exposed to cysteamine or to the $48 \mathrm{hr}$. dialysed lysate lysed most rapidly. The presence of $\mathrm{Co}^{++}$did not accelerate lysis of heated organisms but did significantly hasten the lysis of non-heated organisms with one exception. The single exception was that of the culture filtrate containing cysteamine and it is believed that the addition of $\mathrm{Co}^{++}$to this sample neutralized the cysteamine by forming an inactive chelate. As 
evidence of chelate formation, solutions containing cysteamine and $\mathrm{Co}^{++}$ developed a pink colour. Lysis eventually did occur in these samples presumably because of the excess $\mathrm{Co}^{++}$.

\section{DISCUSSION}

Lytic phenomena in the genus Bacillus have been reported quite frequently within the past 3 years (Greenberg \& Halvorson, 1955; Ivánovics \& Alföldi, 1957; Nomura \& Hosoda, 1956; Norris, 1957; Strange \& Dark, 1957). The present observations are similar to those of the majority of the previous reports in that $(a)$ post-log. phase rather than younger crops of organisms are most readily lysed, $(b)$ there is considerable strain and species specificity, $(c)$ a complex medium usually is required, and $(d)$ most of the formed elements of the organism are destroyed during lysis.

The present observations differ from those of the previous studies on lysis of organisms of Bacillus sp. in that the assay organisms themselves actively participate in the lytic process. Organisms inactivated by heat do not lyse nor are isolated cell walls destroyed by cysteamine. In order to lyse, the assay organisms must be maintained at $37^{\circ}$ in staled broth in the presence of the cysteamine compounds for several hours.

Induction of lysis of strains of Bacillus spp. by the cysteamine compounds is somewhat comparable to induction of lysis of various bacteria of different genera by glycine (Cowles, 1946). With glycine, post-log. phase metabolizing assay organisms are required, and 3-20 hr. are needed for the appearance of visible lysis. As with cysteamine, the cultures do not become completely sterile. Although glycine has a much broader bacteriolytic spectrum than has the cysteamine compounds, it is much weaker in activity. Concentrations of glycine as great as molar are required, whereas in the present experiments, active compounds induced lysis optimally at concentrations as low as $10^{-4} \mathrm{M}$.

An interesting feature of induction of lysis by cysteamine is that post-log. phase organisms which are used as inoculum for fresh cultures do not lyse within 4-8 hr. in the presence of the compound in fresh broth. Rather, the organisms proceed through the normal phases of growth and give rise to progeny which do lyse within 4-8 hr. in the post-log. phase provided cysteamine has not been removed from the environment. Apparently, fresh nutrient broth lacks factors possessed by staled broth that are required for cysteamine lysis. An alternative hypothesis is that fresh broth permits the post-log. phase organisms to begin logarithmic growth before $4 \mathrm{hr}$. has elapsed and they thus temporarily escape the usual fate of post-log. phase organisms exposed to cysteamine.

The requirement for nutrient broth rather than the defined medium for the exhibition of cysteamine activity is analogous to the situation reported by Ivánovies \& Alföldi (1957) in which induction of lysis of Bacillus megaterium by thioglycollate or ultraviolet light occurred only when the organisms were grown in a complex medium. Perhaps cell walls are synthesized differently in complex media and simpler defined nutritive environments (cf. Litwac \& Pramer, 1956). In the present study, however, organisms grown in the 
defined medium did lyse in the presence of cysteamine when they were suspended in staled nutrient broth. It is of interest to note that the intratransguanylation product of AET (i.e. 2-mercaptoethylguanidine) was found to be considerably more active in protecting bone marrow cells from $\mathrm{X}$-irradiation when the cells were suspended in plasma rather than in a chemically defined medium or in saline (Smith, 1957).

Of interest are reports that cystamine is metabolized to a dark coloured compound by extracts of mammalian amine oxidases (Cavallini, De Marco \& Mondovi, 1957; Bergeret \& Blaschko, 1957). In the present study, one of the sensitive and two of the resistant strains of Bacillus spp. likewise produced a dark pigmented compound from cysteamine and from cystamine. Unpublished observations in this laboratory indicate that cysteamine is neither deaminated nor desulphurated by intact organisms or by cell-free extracts of sensitive strains.

Possible mechanisms whereby the cysteamine compounds induce lysis of sensitive strains include the following:

A. Activation of a normal 'lysozyme-like' autolytic enzyme. Autolytic enzyme molecules are present in large amount in post-log. phase organisms of Bacillus spp. (Richmond, 1957; Strange \& Dark, 1957). This enzyme presumably facilitates the emergence of the spore through the vegetative cell wall. In the present study, spores did not develop because of the lack of sufficient $\mathrm{Mn}^{++}$(Weinberg, 1955) but the organisms did possess an autolytic mechanism as demonstrated by their rapid autolysis in the presence of $\mathrm{Co}^{++}$or when heated to $58^{\circ}$. Possible activation of this autolytic mechanism by the cysteamine compounds might be comparable to the activation of egg-white lysozyme by ethylene diamine tetraacetic acid (Repaske, 1956).

B. Suppression of new cell-wall synthesis. If continual synthesis of new cellwall material by post-log. phase organisms is necessary to balance the normal autolytic mechanism, then a suppressor of such synthesis would indirectly cause the organisms to lyse. It is known that cell-wall synthesis may be suppressed by $(a)$ penicillin and $(b)$ a deficiency of a constituent of cell walls such as $\alpha, \epsilon$-diaminopimelic acid in the nutrient for certain auxotrophs (McQuillen, 1958). Unlike penicillin, cysteamine and related compounds are not active during logarithmic growth; moreover, $\mathrm{Co}^{++}$neutralizes rather than enhances these compounds, presumably by formation of an inactive chelate. Conceivably, such compounds, rather than an unknown analogue, might be incorporated into cell walls of post-log. phase organisms of sensitive strains with the result that such walls could not maintain their structural integrity.

C. Binding of metallic ions in cell walls. Kozloff \& Lute (1957) presented evidence that cell walls of Escherichia coli contain zinc atoms. It is possible that metallic ions are present in the walls of Bacillus and that post-log. phase organisms depend on such ions to maintain the structural integrity of the walls to a greater extent than do younger cells. However, numerous metalbinding agents other than cysteamine and its derivatives have been found in this laboratory (unpublished observations) to be unable to induce lysis of post-log. phase organisms of Bacillus spp. 
D. Binding a metallic ion inhibitor of lysis. Autolysis of Streptococcus faecalis has been observed to be suppressed by $\mathrm{Mn}^{++}$(MacLeod, 1951; van Eys \& Pearson, 1954). In the present study, lysates, culture filtrates and fresh nutrient broth gained autolytic activity by being dialysed for 1-2 days. It is conceivable that such dialyses remove metallic ions that are tightly bound to non-dialysable molecules in the preparations, and that such metallic ions are suppressors of autolytic activity of post-log. phase organisms of Bacillus. Thus, dialysed preparations might induce lysis in the absence of, for example, cysteamine, whereas in non-dialysed materials, lysis would occur only if the active compounds herein reported combined with certain metallic ions of these materials.

E. Suppression of a protease that might inhibit lysis. Experiments performed in this laboratory (to be published) indicate that cysteamine and its derivatives suppress the auto-protease activity of extracts of sensitive organisms. If protease activity is needed to inhibit normal autolysis of post-log. phase organisms then a suppressor of such activity would indirectly induce lysis. Obviously much additional investigation is needed to establish the relationship, if any, among the biochemical mechanisms whereby cysteamine and similar compounds protect cells against radiation damage, suppress protease activity, and induce lysis of post-log. phase organisms of sensitive strains of Bacillus.

One of us (E. D. W.) wishes to acknowledge the financial support of the National Science Foundation and the National Institutes of Health. Portions of this work were performed, while one of us (E. D. W.) was the recipient of a Visiting Scientist appointment at the National Institutes of Health.

\section{REFERENCES}

Bergeret, B. \& Blaschko, H. (1957). The oxidation of cystamine and homocystamine by mammalian enzymes. Brit. J. Pharmacol. $12,513$.

Cavaluini, D., De Marco, C. \& Mondovi, B. (1957). Cystaldimine: the product of oxidation of cystamine by diamine-oxidase. Biochim. biophys. Acta, 24, 353.

Cowles, P. B. (1946). The action of glycine on bacterial suspensions. Yale J. Biol. Med. 19, 835.

DyAR, M. T. (1947). A cell wall stain employing a cationic surface-active agent as a mordant. J. Bact. 53, 498.

Greenberg, R. A. \& Halvorson, H. O. (1955). Studies on an autolytic substance produced by an aerobic spore-forming bacterium. J. Bact. 69,45 .

Ivánovics, G. \& ALFöLdI, L. (1957). Bacteriocinogenesis in Bacillus megaterium. J. gen. Microbiol. 16, 522.

KozLoff, L. M. \& Lute, M. (1957). Viral invasion. II. The role of zinc in bacteriophage invasion. J. biol. Chem. 228, 529 .

Litwack, G. \& Pramer, D. (1956). Growth of Micrococcus lysodeikticus as substrate for lysozyme. Proc. Soc. exp. Biol. Med. 91, 290.

MACLEOD, R. A. (1951). Further mineral requirements of Streptococcus faecalis. $J$. Bact. 62, 337.

MCQuILLEN, K. (1958). Lysis resulting from metabolic disturbance. J. gen. Microbiol. $18,498$.

Nomura, M. \& Hosoda, J. (1956). Nature of the primary action of the autolysin of Bacillus subtilis. J. Bact. 72, 573. 
Norris, J. R. (1957). A bacteriolytic principle associated with cultures of Bacillus cereus. J. gen. Microbiol. 16, 1.

REPASKe, R. (1956). Lysis of Gram-negative bacteria by lysozyme. Biochim. biophys. Acta, 22, 189.

Richmond, M. H. (1957). Bacterial lysozyme. J. gen. Microbiol. 16, iv.

Salton, M. R. J. \& HonNe, R. W. (1951). Studies of the bacterial cell wall. II. Methods of preparation and some properties of cell walls. Biochim. biophys. Acta, 7, 177.

Shapira, R., Doherty, D. G. \& Burnett, W. T., jun. (1957). Chemical protection against ionizing radiation. III. Mercaptoalkylguanidines and related isothiuronium compounds with protective activity. Rad. Res. 7, 22.

Sмiтh, L. H. (1957). Protective effect of 2-mercaptoethylguanidine on bone marrow cells X-irradiated in vitro. Exp. Cell Res. 13, 627.

Strange, R. E. \& Dark, F. A. (1957). A cell-wall lytic enzyme associated with spores of Bacillus species. J. gen. Microbiol. 16, 236.

van Eys, J. \& Pearson, W. N. (1954). Effect of manganese and other factors on early autolysis of cultures of Streptococcus faecalis. J. Bact. 68, 362 .

Weinberg, E. D. (1955). The effect of $\mathrm{Mn}^{++}$and antimicrobial drugs on sporulation of Bacillus subtilis in nutrient broth. J. Bact. 70, 289.

Weinberg, E. D. (1957). Lysis of Bacillus subtilis by cystamine. Exp. Cell. Res. 13, 175.

(Received 2 May 1958) 